\title{
Evaluation of Serum Apo B and A-I as Cardiac Biomarkers in Young Adult Population
}

\author{
Authors \\ Jabeen Fatima ${ }^{1}$, Anees Syyeda ${ }^{2}$, Sudha Bala ${ }^{3}$ \\ ${ }^{1}$ Resident, Department of Biochemistry, Deccan College of Medical Sciences, Hyderabad \\ ${ }^{2}$ Professor, Department of Biochemistry, Deccan College of Medical Sciences, Hyderabad \\ ${ }^{3}$ Assistant Professor, Department of SPM, Deccan College of Medical Sciences, Hyderabad \\ Corresponding Author
}

Dr Anees Syyeda

Professor, Department of Biochemistry,

Deccan College of Medical Sciences, Hyderabad (Telangana, India)

Email: syyeda69@hotmail.com

\begin{abstract}
Clinically normal young adults with no well-known risk factors may present with metabolic syndrome, hypertension, atherogenic dyslipidemia and type-II diabetes. Age and sex are two non-modifiable risk factors for developing cardiovascular disturbances which may affect the levels of apolipoprotein $B$ and apolipoprotein A-I.

The objective is to study serum apo B and A-I levels in association with the conventional lipid profile parameters in atherogenic dyslipidemia.

In this hospital-based prospective study, clinically normal males $(n=172)$ and females $(n=188)$, aged between 20-39 years, divided into two age groups: group-I (20-29 years) and group-II (30-39 years), subjected to overnight fasting had their lipid profile parameters (enzymatic colorimetric assay) along with the serum levels of $а$ apoB and apoA-I (immunoturbidimetry method) estimated. Subjects with body mass index (BMI) >30 were excluded from the study. Data was statistically analysed using Chi-square test and ROC curves.

ApoB levels showed continuous increase with age in both sexes with males having significantly raised $(p<0.01)$ levels in both groups compared to females. ApoA-I levels declined with increasing age in both sexes and this decline was relatively earlier in males. Within each group females were having relatively raised $(p<0.05)$ apoA-I levels than males. Moderate to strong correlations were observed between serum apoA-I and HDL-C concentrations $(p<0.0001)$ and between serum apoB and LDL-C concentrations $(p<0.0001)$, respectively.

$A p o B$ may be a more sensitive measure of atherogenic dyslipidemia than LDL-C and a better index for assessing cardiovascular risk, whereas apoA-I did not add significantly to the estimation of future atherosclerotic vascular risk.

Keywords- ApoA-I, ApoB, Cardiovascular risk, Dyslipidemia, Lipid profile.
\end{abstract}




\section{INTRODUCTION}

During the last several years interest has focussed on the importance of the lipid transporting apolipoproteins. Apolipoproteins are the protein components of macromolecular lipoprotein complexes. One or more apolipoproteins are present in each lipoprotein. Some apolipoproteins are integral and cannot be removed (apoB and apoA), whereas others are bound to the surface and are free to transport to other lipoproteins (apoC and apoE). Apo B, the structural protein for the atherogenic lipoproteins, very low-density lipoproteins (VLDL), intermediate-density lipoproteins (IDL), and both large, buoyant LDL and small dense LDL, is responsible for transporting lipid from liver and gut to peripheral tissues. Each lipoprotein particle contains 1 apoB molecule; therefore, the total apoB level corresponds to the total number of atherogenic particles and denotes the atherogenic potential of the whole lipoprotein fraction. By contrast, apoA-I is the major structural protein for high-density lipoprotein (HDL), and reflects the atheroprotective side of the lipid metabolism. ApoA-I is produced both in the liver and intestine and is responsible for initiating reverse cholesterol transport, whereby excess cholesterol in peripheral tissues is carried back to the liver for excretion.

Atherosclerosis of the vascular system is the basis of wide spectrum of clinical outcomes like coronary artery disease (CAD), cerebrovascular disease and peripheral vascular occlusive disease. CAD is the leading cause of morbidity and mortality in both developed and developing countries. CAD epidemic in India has entered into an epidemiological transition phase. The prevalence of CAD in urban India $(10 \%)$ is double than that of rural India (5\%) and about 4 -fold higher than in the US $(2.5 \%) .{ }^{[1]}$ The prevalence rates of CAD appear to be higher in South India. ${ }^{[2]}$ The escalating epidemic of CAD in India is due to the combination of genetic predisposition and lifestyle changes. ${ }^{[3,4]}$ Atherosclerotic vascular disease is the major cause of morbidity and mortality from middle age onwards. ${ }^{[5]}$
Dyslipidemia is considered to be the forerunner of atherosclerotic vascular disease. Adult Treatment Panel (ATP) III guidelines ${ }^{[6]}$ are widely practiced to identify the dyslipidemia. Current ATP III guidelines recommend lipid screening in all adults $>20$ years. The screen should include a fasting lipid profile (Total cholesterol [TC], Triglycerides [TG], HDL-c and LDL-c) repeated every 5 years. But this approach cannot find out all individuals at the risk of developing atherosclerotic vascular disease.

Clinically normal young adults (20-39 years) with no well-known risk factors may present with metabolic syndrome, hypertension, atherogenic dyslipidemia and type-II diabetes. ${ }^{[7]}$ Age and sex are two non-modifiable risk factors for developing cardiovascular disturbances which may affect the serum levels of apolipoproteins B and A-I. ${ }^{[8]}$

Within a population, as the levels of cholesterol rise, the risk of coronary artery disease also increases. But only a small portion of population has very high levels of cholesterol. A major finding of Framingham study ${ }^{[9]}$ is that most cases of premature vascular disease occurred in individuals with total and LDL cholesterol levels that were indistinguishable from those who did not develop premature disease. But at the same time, elevated LDL-c is the type of dyslipidemia most closely associated with coronary artery disease. These two contradictory observations point towards the necessity of concentrating on the non-lipid part of the lipoproteins, namely apoproteins. Since LDL-c is considered to be more linked to the atherosclerotic disease, it is better to study the apoprotein of LDL-c i.e., apolipoproteinB.

Dyslipidemia, high apoB and low apoA-I are widely accepted as the risk factors for CAD in subjects with well-known risk factors and the aged. In this context, this study attempts to find out the role of apo B and A-I against the routinely tested lipid parameters in clinically normal young adult population, as potential cardiac biomarkers for risk assessment. 


\section{MATERIALS AND METHODS}

This hospital-based prospective study was carried out at Owaisi Hospital and Research Centre, attached to Deccan College of Medical Sciences, Hyderabad, India, over a period of 18 months, from November 2014 to April 2016. Convenient (simple random) sampling has been adopted for effective cost-control. A total of 360 clinically normal adults, aged between 20-39 years, presenting to the outpatient department for general health check-up, were taken up for this study. There was no past history of any known disease or illness in the last 6 months. Subjects with BMI $\geq 30$ and/or on lipidaltering medications were excluded from this study. This study population was divided into two groups based on their age. Group-I $(n=164)$ included adults aged between 20-29 years and Group-II $(n=196)$ included adults aged between 30-39 years.

As per the selection criteria in both groups, subjects were included in the study only after obtaining their informed consent. This study was approved by the Medical Ethics Committee of this institution.

Biochemical analysis: Under strict aseptic conditions, $5 \mathrm{~mL}$ of fasting blood samples were withdrawn from antecubital veins into plain vacutainers $(75 \mathrm{~mm} \times 13 \mathrm{~mm})$ and centrifuged at $3000 \mathrm{rpm}$ for 10 minutes and sera obtained for analysis. Fully automated analyser cobas c311 by Roche/Hitachi was used for the estimation of apolipoprotein B and A-I (immunoturbidimetric assay), ${ }^{[10-12]}$ TC (CHOD-POD photometric assay), TG, HDL-c, LDL-c (homogenous enzymatic colorimetric assay) and VLDL-c (VLDL=TG/5), using kits provided by cobas.

All the analytes estimated are subjected to standard quality control (QC) guidelines. The clinical biochemistry laboratory of Owaisi Hospital and Research Centre is a participant of External Quality Assurance Scheme (EQAS) from CMC Vellore. Internal Quality Assessment is run twice daily (12hourly) with both first party controls (cobas- PCC1, PCC2) and third party controls (Randox).

Statistical analysis: All the data so collected was duly recorded and compiled; results and observations drawn and statistically analysed using
MS Excel 2007 and Epi Info Windows software. Values were expressed as mean \pm standard deviation (SD). Chi-square test was used to get the statistical significance. $\mathrm{p}$ value $<0.05$ was considered statistically significant at the level of $95 \%$ confidence limit $(\mathrm{Cl})$. Receiver Operating Characteristic (ROC) curve was drawn to calculate the sensitivity and specificity of apoB and apoA-I for atherogenic dyslipidemia.

The currently used ATP III guidelines was used to select the cut-off values of TC (>200 mg/dL), TG (>150 mg/dL), HDL-c (<40 mg/dL), LDL-c (>100 $\mathrm{mg} / \mathrm{dL})$. BMI was calculated as weight $(\mathrm{kg})$ divided by squared height (metre).

\section{RESULTS}

The present study aims at analysing the serum apolipoprotein B and A-I levels in young adults, their relationship with the conventional lipid profile and determining the sensitivity and specificity of apoB and apoA-I with respect to LDL-c and HDL-c as better markers of atherogenic dyslipidemia.

Table 1 shows the baseline characteristics of the total 360 subjects studied. Group-I $(n=164)$ comprised of 78 males and 86 females, with mean age $25.78 \pm 2.69$ years and mean BMI $26 \pm 3 \mathrm{~kg} / \mathrm{m}^{2}$. Group-II $(n=196)$ comprised of 94 males and 102 females, with mean age $34.54 \pm 2.93$ years and mean BMI $27 \pm 4 \mathrm{~kg} / \mathrm{m}^{2}$. Both the groups have a same sex distribution ratio with no significant differences ( $\mathrm{p}>0.05$ ). (Figure.1)

Table 2 shows mean values with SD of all parameters assessed in both groups with test of statistical significance. Serum apoB levels tend to increase continuously with increasing age in both the sexes. But the increase in serum apoB levels in males in both groups was relatively more as compared to females leading to significant $(\mathrm{p}<0.01)$ sex related differences in apoB levels. Age related variations in serum apoA-I levels were not similar to that of serum apoB levels. Serum apoA-I showed decline in levels with increasing age in both the sexes. In both age groups females were having significantly raised $(\mathrm{p}<0.05)$ serum apoA-I levels as compared to males. (Figure.2) 
The comparison on the basis of lipid profile parameters demonstrated that there was not much significant difference between TC $(\mathrm{p}>0.05)$, TG $(p>0.05)$ and VLDL-c ( $>0.05)$. The mean serum values of TC of both the groups were in the borderline range $(200-240 \mathrm{mg} / \mathrm{dL})$, whereas, TG values were within the desirable level of $<150$ $\mathrm{mg} / \mathrm{dL}$. Significant difference was seen in HDL-c $(p<0.01)$ and LDL-c $(p<0.01)$ levels between the two groups. Serum HDL-c levels of both the groups were within the optimal range $(40-59 \mathrm{mg} / \mathrm{dL})$ and LDL-c levels of group I and II were within the optimal $(<100 \mathrm{mg} / \mathrm{dL})$ near optimal (100-130 $\mathrm{mg} / \mathrm{dL}$ ) ranges respectively. (Figure.3)

The Receiver Operating Characteristic (ROC) curves were drawn for apoB and apoA-I. (Fig.4) The cut-off values for apoB and apoA-I were determined as $98.5 \mathrm{mg} / \mathrm{dL}$ and $148.5 \mathrm{mg} / \mathrm{dL}$ respectively. The sensitivity value of apoB maximally correlated with the area of true-positive than the areas of false-positive and false-negative in the graph. Therefore, the sensitivity score for apoB was positive. In this study, subjects had shown maximum sensitivity for apoB (AUC: $0.737 ; 95 \%$ $\mathrm{Cl}$ : 0.682-0.792) which was highly significant $(\mathrm{p}<0.0001)$. Moreover, apoA-I was poorly correlated with the area of true-positive than the areas of false-positive and false-negative (AUC: 0.320; 95\% Cl: 0.264-0.375) with high significance value $(\mathrm{p}<0.0001)$. Hence, the ROC curve concludes that, apoB is a more sensitive marker for assessing cardiovascular risk than LDL-c, but the same cannot be said for apoA-I when compared to HDL-c.

Table 1. Baseline characteristics of study subjects $(n=360)$ showing sex distribution and mean \pm SD for age and BMI.

\begin{tabular}{|l|l|l|}
\hline & Group-I $(\mathrm{n}=164)$ & Group-II $(\mathrm{n}=196)$ \\
\hline Males $(48 \%)$ & $\mathbf{7 8}$ & $\mathbf{9 4}$ \\
\hline Females $(52 \%)$ & $\mathbf{8 6}$ & $\mathbf{1 0 2}$ \\
\hline Age (years) & $\mathbf{2 5 . 7 8} \pm \mathbf{2 . 6 9}$ & $\mathbf{3 4 . 5 4 \pm 2 . 9 3}$ \\
\hline BMI $\left(\mathrm{kg} / \mathrm{m}^{2}\right)$ & $\mathbf{2 6} \pm \mathbf{3}$ & $\mathbf{2 7} \pm \mathbf{4}$ \\
\hline
\end{tabular}

Table 2. Mean levels (mean \pm SD) of apoB, apoA-I and lipid profile in baseline serum samples of groups I and II.

\begin{tabular}{|l|l|l|l|}
\hline $\begin{array}{l}\text { Parameters } \\
(\mathrm{mg} / \mathrm{dL})\end{array}$ & $\begin{array}{l}\text { Group-I } \\
(\mathrm{n}=164)\end{array}$ & $\begin{array}{l}\text { Group-II } \\
(\mathrm{n}=196)\end{array}$ & $\begin{array}{l}\mathrm{p} \\
\text { value }\end{array}$ \\
\hline ApoB & $95.18 \pm 11.23$ & $100.41 \pm 18.44$ & 0.001 \\
\hline ApoA-I & $142.43 \pm 16.23$ & $137.73 \pm 12.38$ & 0.002 \\
\hline TC & $214.56 \pm 40.73$ & $223.41 \pm 52.08$ & 0.077 \\
\hline TG & $122.46 \pm 25.51$ & $128.14 \pm 31.98$ & 0.067 \\
\hline HDL-c & $43.64 \pm 6.60$ & $40.82 \pm 10.52$ & 0.003 \\
\hline LDL-c & $88.6 \pm 26.25$ & $105.16 \pm 31.07$ & 0.008 \\
\hline VLDL-c & $22.88 \pm 12.65$ & $25.38 \pm 14.98$ & 0.091 \\
\hline
\end{tabular}

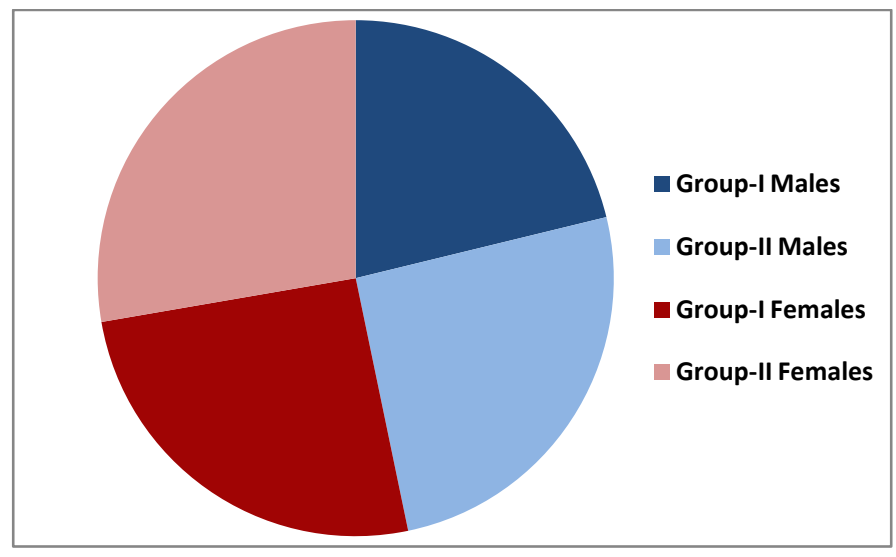

Figure 1. Graphical representation of percentage distribution of gender of groups I and II.

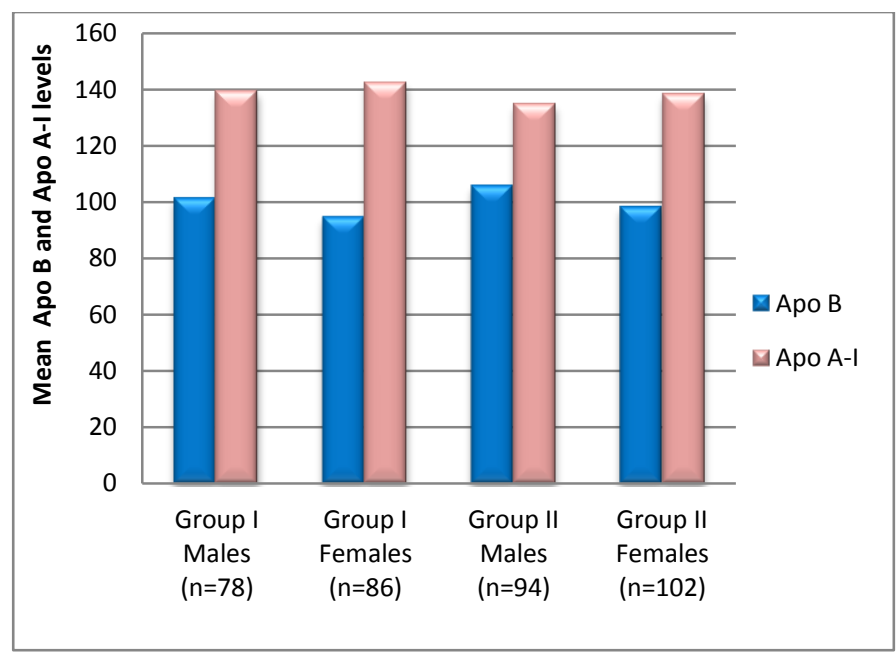

Figure 2. Graphical representation of mean serum values of apoB and apoA-I in males and females of Group I and II. 


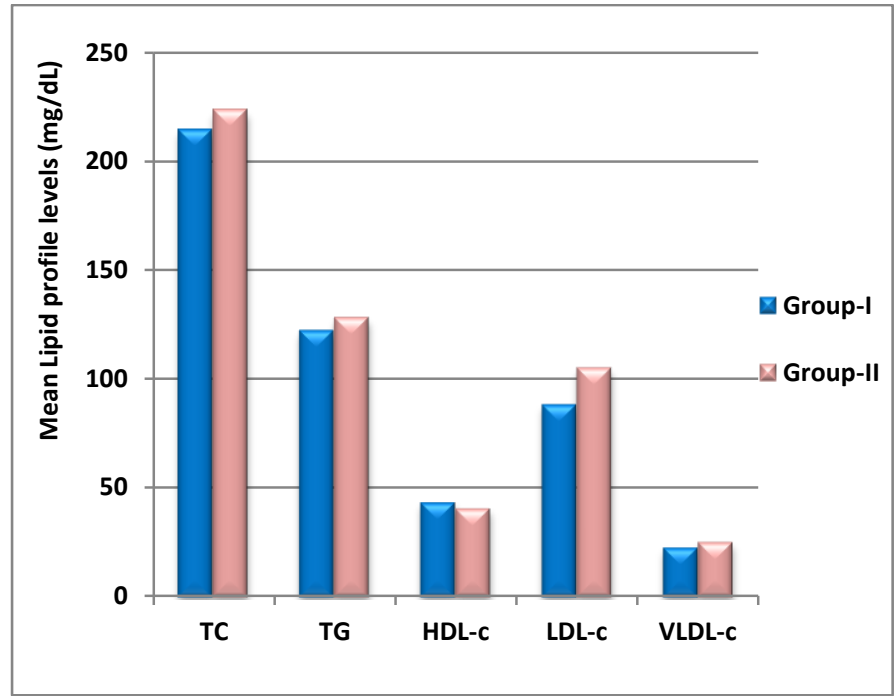

Figure 3. Graphical representation of mean values of serum lipid profile parameters in Group I and II.

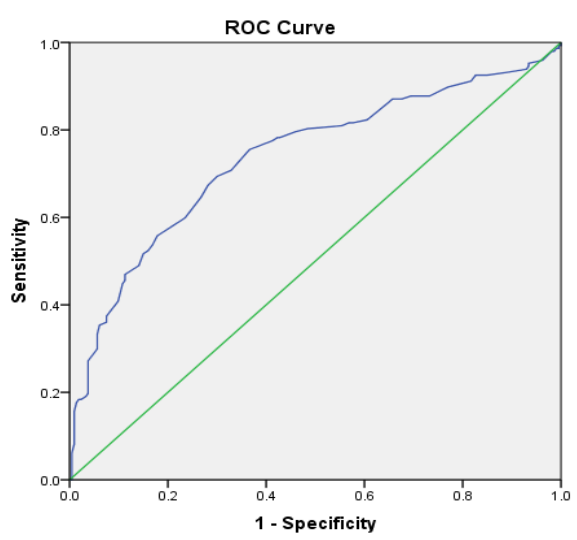

(a)

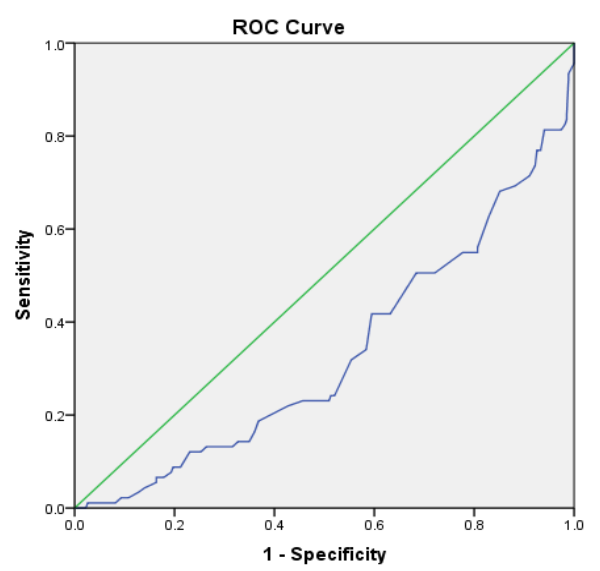

(b)

Figure 4. (a) The ROC curve of apoB (AUC: 0.737; standard error: $0.027 ; 95 \% \mathrm{Cl}: 0.682-0.792$ and significance level $\mathrm{p}<0.0001$ ) (b) The ROC curve of apoA-I (AUC: 0.330; standard error: 0.033; 95\% Cl: $0.266-0.375$ and significance level $\mathrm{p}<0.0001$ )

\section{DICUSSION}

Apoipoprotein B is a constituent component of LDL and apolipoprotein A-I of HDL. Recently they have been proposed as better markers to assess the risk of CAD. Some studies suggested the role of apolipoproteins in $\mathrm{CAD}$, but the conclusions drawn are variable and none of the studies, to the best of our knowledge, were carried in young adult population in specific. Age and sex are two nonmodifiable risk factors for developing atherogenic dyslipidemia and eventually CAD, which may affect serum apolipoprotein levels. Data regarding the effect of age and sex on serum apoB and apoA-I levels is very limited in the Indian population. The uncertainties are limiting the use of these apolipoproteins in routine diagnosis, which may otherwise prove beneficial in screening populations having high incidence of CAD. To investigate their role in $\mathrm{CAD}$, age and sex related variations need to be studied. In this study, serum apo B levels showed a continuous increase with age in both sexes, which may indicate towards a physiological increase in risk of CAD with advancing age. This risk seems to be more pronounced in males, having increased number of atherogenic particles (apoB) and decreased number of antiatherogenic particles (apo A-I). On the contrary, serum apoA-I levels slightly declined with increase in age with females of both groups having significantly higher values than males. Very similar results were reported in Indian, ${ }^{[8]}$ Swedish $^{[13]}$ and Mexican ${ }^{[14]}$ population studies. Age groups taken in these studies were different from the present study but that did not affect the results. In the Mexican population study, relatively lower mean levels of serum apo B and A-I were reported as compared to the results of the present study. This strengthens the need to obtain more data regarding these apolipoproteins in population-based studies worldwide.

In this study, the significant variable associated with atherosclerotic vascular disease was found to be high values of apoB ( $\geq 98.5 \mathrm{mg} / \mathrm{dL})(\mathrm{p}<0.0001)$. The close association of apoB with atherosclerotic vascular disease is supported by Quebec Cardiovascular study, ${ }^{[15]}$ which showed that apoB 
was associated with CAD, independent of LDL. A prior study ${ }^{[16]}$ demonstrated that use of single index, namely apoB, is as efficient for categorization and follow-up of patients with dyslipidemia as the currently used lipid profile. Another study ${ }^{[17]}$ corroborated the lack of a lipid-independent association between apoB levels and CAD. That suggests that a mere elevation of apoB cannot produce atherosclerosis even though the modified apoB could initiate the plaque formation. It is because the pathophysiology of atherosclerosis involves initiation by oxidatively modified residues of apoB and progression of the lesion requires subintimal deposition of lipids. Constitutively synthesized apoB is degraded by the proteasome as and when synthesized, if there are no enough lipids available to form lipoprotein complexes. ${ }^{[18]}$

In current study, the rationale for using apoB and AI for risk assessment for $\mathrm{CAD}$ is more directly related to the number and size of circulating atherogenic particles than to the concentration of cholesterol in these particles. Although LDL-c may not be typically elevated in dyslipidemia, it is highly atherogenic because of the increased presence of small, dense LDL particles. Therefore, measuring only the LDL-c can underestimate the atherogenic burden. Measurements of apoB are more accurate, as they quantify the total number of potentially atherogenic particles in plasma. The finding of elevated apoB in a person with normal LDL-c level, likely indicates an increased cardiovascular risk. ApoB may also be a more important index than LDL-c in patients with atherogenic dyslipidemia, since it is disproportionately higher in patients with high TG levels. Apolipoprotein B being a protein unlike lipid variables, the assay of apoB can be performed on non-fasting blood samples and this is definitely an added advantage over the routine lipid profile which requires a fasting blood sample. ${ }^{[19]}$

By contrast, patients with low levels of apoA-I $(<120 \mathrm{mg} / \mathrm{dL})$ are more likely to have CAD than those with high apoA-I levels (>160 mg/dL). As with LDL, the cholesterol content of HDL particles varies across patient types and is influenced by plasma TG levels; patients with high TG tend to have low apoA-I levels. Nonetheless, it is unclear whether apoA-I alone is a predictor of cardiovascular risk independent of its association with HDL-c. A number of population-based studies have shown that apoA-I provided little or no additional predictive value compared with the conventional parameters and was not as strong a predictor as apoB.

The risk attributed to TC and LDL-c is not linear and increases sharply over the higher ranges. Reduction of serum cholesterol is important in reducing the risk of CAD but cholesterol and other lipids are poor screening tests for risk evaluation of atherosclerotic vascular disease. ${ }^{[20]}$ The reason for this discrepancy is that the screening potential of a factor depends not only on the power of its relationship with disease but also on its variation with magnitude across individuals in a community. Moreover, multifactorial nature of the disease creates complication in the selection of a single screening tool.

\section{CONCLUSION}

Based on the finding of current study and previous studies, suggesting that risk of CAD is increasing almost linearly with increasing values of apoB, it seems logical to add apoB into clinical practice as a simple, robust, accurate risk indicator of great value in health screening.

Serum apoB concentration, which reflects the number of small, dense LDL-c particles, may prove to be a more sensitive marker of atherogenic dyslipidemia than LDL-c and a better index for assessing cardiovascular disease risk.

The protective effect offered by HDL-c is attributable to the unique apolipoprotein $\mathrm{f}$ HDL-c, apolipoprotein A-I. But in this study apoA-I does not seem to add significantly to the estimation of future atherosclerotic vascular risk. Further research is required on larger sample sizes to find out the role of apoA-I as a cardiac biomarker, independent of the HDL-c. 


\section{ACKNOWLEDGEMENT}

The kind support from Deccan College of Medical Sciences for carrying out the work and Owaisi Hospital and Research Centre for recruitment of subjects is acknowledged.

\section{REFERENCES}

1. Reddy KS, Rising burden of cardiovascular diseases in India. Cardiology Society of India 1998:63-72.

2. Enas EA, Yusuf S, Sharma S, Coronary artery disease in South Indians: second meeting of the International Working Group, March 16, 1997, Anaheim, Carlifornia. Indian Heart Journal 1998;50:105-13.

3. Enas EA, Yusuf S, Third Meeting on the International Working Group on Coronary Artery Disease in South Asians, March 29,1998, AtlantaGA, USA. Indian Heart Journal 1999;51:99-103.

4. Ross R, Atherosclerosis- an inflammatory disease. New England Journal of Medicine 1999;340:115-126.

5. Fuster V, Ross R, Topol EJ, eds, Atherosclerosis and coronary artery disease. Vol 1 and 2 Philadelphia: Lippincott Raven, 1996.

6. Executive summary of third report of the National Cholesterol Education Program (NCEP) Expert Panel on Detection, Evaluation, and Treatment of High Blood Cholesterol in Adults (Adult Treatment Panel III). JAMA, 2001;285:2486-2497.

7. A Onat, G Can, G Hergenc, M Yazici, A Karabulut and S Albayrak, Serum apolipoprotein $B$ predicts dyslipidemia, metabolic syndrome and, in women, hypertension and diabetes, independent of markers of central obesity and inflammation, International Journal of Obesity 2007;31:1119-11125.

8. R Sharma, B Singh, M Mahajan, R Kant, Age and Sex: important determinants in affecting the levels of serum apo $B$ and $A-I$ in Indian population. The Internet Journal of Cardiology 2006;4:1.

9. Kannel WB, Castelli WP, Gordon T, Cholesterol in the prediction of atherosclerotic disease. New perspectives based on Framingham study. Annals of Internal Medicine 1979;90:85-91.

10. Siedel J, Schiefer S, Rosseneu M, et al. Immunoturbidimetric Method for Routine Determinations of Apolipoproteins A-I, A-II and $B$ in Normo and Hyperlipemic Sera compared with Immunonephelometry. Clinical Chemistry. 1988;34(9):1821-1825.

11. Rifai N, King ME. Immunoturbidimetric Assays of Apolipoproteins A, AI, AII, and B in serum. Clinical chemistry. 1986;32:957961.

12. Naito HK. Reliability of Lipid, Lipoprotein and Apolipoprotein Measurements. Clinical Chemistry. 1988;34:B84-B94.

13. Jungner I, Marcovina SM, Walldius G, Holme I, Kolar $\mathrm{W}$ and Steiner E, Apolipoprotein B and A-I value in 147576 Swedish males and females, standardized according to the world health organizationInternational Federation of Clinical First International Reference Materials. Clinical Chemistry 1998;44:1641-49.

14. Valles V, Aguilar-Salinas CA, Gomez-Perez FJ, Rojas R, Franco A, Olaiz G et al., Apolipoprotein $B$ and A-I distribution in Mexican Urban Adults: Results of a Nationwide survey. Metabolism 2002; 51:560-68.

15. Lamarche B, Tchernof A, Moorjani S, Cantin B, Dagenais GR, Lupien PJ et al., Small dense low density lipoprotein particles as a predictor of the risk of ischemic heart disease in men. Prospective results from Quebec Cardiovascular study. Circulation 1997;95:69-75.

16. Saman Miremadi, Allan Sniderman and Jiri Frohlich, Can Measurement of Serum Apolipoprotein replace the lipid profile monitoring of patients with lipoprotein 
disorders? Clinical Chemistry 2002;48: 3484-88.

17. Sweetnam PM, Bolton $\mathrm{CH}$, Downs L, Durrington PN, Mackness MI, Elwood PC et al. Apolipoproteins AI, AII, and B, lipoprotein (a) and the risk of ischemic heart disease. Caerphilly study. European Journal of Clinical Investigations 2000;30:947-56.

18. Fisher E et al., Apoprotein B 100 an atypical secretory protein, can be degraded by cytosolic pathway involving heat shock protein 70 and proteasomes. Journal of Biological Chemistry 1997;272:2042720434.

19. Connelly PW, Poapst M, Davingnon J, Lussier-Cacan S, Reeder B, Lessard R et al., Reference values of plasma apolipoproteins $A-I$ and $B$, and association with nonlipid risk factors in the population of two Canadian provinces, Quebec and Saskatchewan. Canadian Heart Health Surveys Research Group. Canadian Journal of Cardiology 1999;40:586-92.

20. Pfeffer MA, Sacks FM, Moye LA et al. Influence of baseline lipids on effectiveness of pravastatin in the cholesterol and recurrent events (CARE) Trial. American College of Cardiology 1993;33:125-130. 